\title{
Supra-threshold scaling, temporal summation, and after-sensation: relationships to each other and anxiety/fear
}

\author{
This article was published in the following Dove Press journal: \\ Journal of Pain Research \\ 27 March 2010 \\ Number of times this article has been viewed
}

\author{
Michael E Robinson' \\ Joel E Bialosky² \\ Mark D Bishop ${ }^{2}$ \\ Donald D Price ${ }^{3}$ \\ Steven Z George ${ }^{2}$ \\ 'Department of Clinical and Health \\ Psychology, University of Florida, \\ Gainesville, FL, USA; ${ }^{2}$ Department \\ of Physical Therapy, University \\ of Florida, Gainesville, FL, USA; \\ ${ }^{3}$ Dentistry and Neurosciences, \\ University of Florida, Gainesville, \\ FL, USA
}

\begin{abstract}
This study investigated the relationship of thermal pain testing from three types of quantitative sensory testing (ie, supra-threshold stimulus response scaling, temporal summation, and after-sensation) at three anatomical sites (ie, upper extremity, lower extremity, and trunk). Pain ratings from these procedures were also compared with common psychological measures previously shown to be related to experimental pain responses and consistent with fear-avoidance models of pain. Results indicated that supra-threshold stimulus response scaling, temporal summation, and after-sensation, were significantly related to each other. The site of stimulation was also an important factor, with the trunk site showing the highest sensitivity in all three quantitative sensory testing procedures. Supra-threshold response measures were highly related to measures of fear of pain and anxiety sensitivity for all stimulation sites. For temporal summation and after-sensation, only the trunk site was significantly related to anxiety sensitivity, and fear of pain, respectively. Results suggest the importance of considering site of stimulation when designing and comparing studies. Furthermore, psychological influence on quantitative sensory testing is also of importance when designing and comparing studies. Although there was some variation by site of stimulation, fear of pain and anxiety sensitivity had consistent influences on pain ratings.
\end{abstract}

Keywords: experimental pain, temporal summation, after-sensation, fear/avoidance, anxiety

\section{Introduction}

Quantitative sensory testing (QST) to determine experimental pain sensitivity for healthy participants and participants with pain conditions appears to be gaining in popularity. ${ }^{1}$ Recent reports have suggested standardized QST protocols for the assessment of individuals with chronic pain conditions. ${ }^{2-4}$ Most QST clinical applications are for the assessment of threshold (the time, or level of stimulation first perceived as painful), supra-threshold (ratings of painful stimulation above threshold), temporal summation, or after-sensation. ${ }^{4}$ Temporal summation refers to the increase in pain perception with repeated stimulation (inter-stimulus interval three seconds or less) that is believed to be related to C-fiber mediated dorsal horn central sensitization. ${ }^{5}$ This phenomenon has been reported to be related to the development of chronic pain conditions. ${ }^{5}$ After-sensation is the heightened level of pain that lingers following the cessation of pain stimulation. ${ }^{5}$ After-sensation has been shown to predict clinical pain ratings, and may be related to endogenous, pain inhibitory mechanisms of C-fiber activity. ${ }^{6}$

Studies involving participants with pain conditions have suggested the importance of QST for supra-threshold testing and assessment of temporal summation. ${ }^{5,7,8}$
Correspondence: Michael E Robinson University of Florida, PO Box 100165 , Gainesville, FL 32610, USA

$\mathrm{Tel}+\mathrm{I} 352.2736 .153$

Fax +I 352.2736.156

Email merobin@ufl.edu 
Previous work has also shown that after-sensation is related to clinical pain report suggesting not only large group differences between patients and controls, but also a relationship between QST, clinical pain ratings, and psychological factors. ${ }^{5,6}$ However, few studies have investigated associations between different stimulus parameters and the influence of anatomical location of stimulus site for classic QST measures. ${ }^{9}$ The above-referenced evidence of the importance of the assessment of central sensitization-related phenomena indicates a need to investigate these parameters for suprathreshold response procedures, temporal summation, and after-sensation at multiple sites along the neural axis.

In summary, studies employing QST methods have used a variety of body sites and stimulus parameters and rarely have multiple measures been compared to each other. This lack of direct comparison across site and test type makes different studies difficult to interpret. Furthermore, the relative contribution of common psychological factors as they relate to supra-threshold response, temporal summation, and after-sensation also needs investigation. The purpose of the present study was to investigate the differences in pain report from three anatomical sites on the body, including the lower extremity, trunk, and upper extremity for supra-threshold response, temporal summation, and after-sensation. Reporting the inter-relationships of the various QST methods of pain induction is important for the interpretation of data across studies, and may serve as a preliminary assessment of the construct validity of these measures.

The analyses of these relationships were primarily for descriptive and exploratory purposes, although our default hypothesis was that the QST measures would all be significantly correlated with each other and that responses would be similar for each of the anatomical sites. We further hypothesized that the measures associated with C-fiber mediated processes (temporal summation and after-sensation) would be more strongly correlated in comparison to A-delta fiber mediated processes (supra-threshold response). We also sought to provide novel information of the relationship of these particular tests with measures of fear of pain, anxiety, anxiety sensitivity, and pain catastrophizing. These particular psychological factors have been related to pain in both clinical and experimental settings ${ }^{10,11}$ and are relevant to fear avoidance models of musculoskeletal pain, which has an important influence on clinical pain conditions. ${ }^{12,13} \mathrm{We}$ hypothesized that fear of pain, anxiety, and pain catastrophizing would be positively associated with the different QST measures.

\section{Materials and methods}

\section{Participants}

A convenience sample of 120 participants was pooled from two previously reported studies that focused on thermal pain responses. ${ }^{14,15}$ Pooling these studies was appropriate for addressing our purposes because the studies had the same eligibility criteria and QST testing procedures. Furthermore, the thermal pain sensitivity data have not been previously used to test the hypotheses reported in this planned secondary analysis. Each study had Institutional Review Board approval and all participants provided informed consent before being included in the studies.

Participants provided demographic information and completed validated psychological questionnaires that were consistent with fear-avoidance models of pain perception. ${ }^{12}$ Participants also completed a visual analog scale (VAS) for fear and anxiety ratings for the current stimuli they were about to experience. Participants then underwent standardized QST for thermal pain sensitivity at the lower extremity, trunk, and upper extremity. The QST protocol as has been employed in previous studies $^{8,15}$ and is further described below.

\section{Psychological measures}

The Anxiety Sensitivity Index (ASI) uses a 16-item, 4-point rating scale to assess anxiety sensitivity, which is the perception of whether experiencing symptoms of anxiety causes harm. The ASI has been validated in community samples ${ }^{16}$ and demonstrated factor invariance across different sex and age groups. ${ }^{17}$

The State-Trait Anxiety Questionnaire (STAI) uses a 40-item, 4-point rating scale to assess dispositional (trait) and situational (state) anxiety symptoms. ${ }^{18}$ We reported the state portion of the STAI as this construct better matched the purposes of this study.

The Coping Strategies Questionnaire (CSQ) uses a 27-item, 7-point rating scale to measures the frequency of use for common pain coping strategies. ${ }^{19}$ We used the catastrophizing subscale that measures helplessness and pessimistic cognitions related to pain perception. The validity of this particular subscale has been supported ${ }^{19,20}$ and the currently recommended scoring system was used in this study. ${ }^{20}$

The Fear of Pain Questionnaire (FPQ-III) uses a 30-item, 5-point rating scale to measure fear about specific situations that would normally produce pain. ${ }^{21}$ The FPQ-III is a commonly used and well-validated instrument that is appropriate for use in nonclinical and clinical populations. ${ }^{21-23}$ 


\section{Quantitative sensory testing}

Thermal stimuli were delivered with the Medoc Neurosensory Analyzer (TSA 2001; Ramat Yishai, Israel). Participants underwent a practice session in which threshold stimuli were rated with a numerical rating scale (NRS) ranging from 0 (no pain) to 100 (worst pain imaginable). After the practice session, pain sensitivity was assessed at the lower-extremity, trunk, and upper-extremity using a counter-balanced testing schedule.

Supra-threshold responses are believed to be representative of A-delta fiber mediated pain sensitivity and these responses were assessed at the volar forearm, posterior superior iliac spine, and dorsal calf via a ramp and hold protocol. The ramp and hold protocol consisted of four heat pulses each lasting five seconds and delivered five seconds from the end of the preceding pulse. Baseline temperature for each heat pulse was $35^{\circ} \mathrm{C}$ and increased at a rate of $10^{\circ} \mathrm{C}$ per second to a randomly determined end point of $45^{\circ} \mathrm{C}, 47^{\circ} \mathrm{C}, 49^{\circ} \mathrm{C}$, or $51^{\circ} \mathrm{C}$. The protocol was repeated twice and NRS ratings were recorded in response to these four temperatures.

Temporal summation and after-sensation are considered to be representative of C-fiber mediated pain sensitivity and these responses were assessed on the palmar surface of the hand, posterior superior iliac spine, and plantar surface of the foot. For temporal summation a train of ten consecutive heat pulses with baseline temperature of $41^{\circ} \mathrm{C}$ and peak temperature of $51^{\circ} \mathrm{C}$ was provided at an inter- stimulus frequently of $0.33 \mathrm{~Hz}$. Participants rated each pulse using the NRS and the fifth pulse was used as the measure of temporal summation. The rationale for choosing the fifth pulse is that it has been demonstrated that "first pain" is either absent or nearly completely diminished after four heat pulses and the remaining pain is delayed by an extent that can only be accounted for by conduction of impulses in C-fibers. ${ }^{24,25}$ This delayed pain is also much greater in magnitude than that evoked by the first pulse in a train of 4-6 pulses or by a single pulse $\mathrm{e}^{24,26,27}$ and therefore is considered to represent a simple measure of temporal summation. ${ }^{25}$ For after-sensations, NRS (numeric rating scale) ratings were collected at 15 seconds following the last stimulus pulse, similar to our previous work. ${ }^{28}$

\section{Data analysis}

Descriptive statistics were generated for the sample, for demographic, psychological, and QST measures. Associations between supra-threshold response, temporal summation, and after-sensation were investigated by Pearson correlation. The effect of the stimulation site was investigated by repeated measures ANOVA, with separate models for supra-threshold response, temporal summation, and after-sensation. Finally, psychological relationships to pain measures were investigated with Pearson correlation and multiple regression. Separate regression models included the fear of pain, anxiety, and pain catastrophizing measures as predictors and suprathreshold response, temporal summation, and after-sensations as the dependent variable.

\section{Results}

Descriptive data for the participants is summarized in Table 1. To reduce the total number of analyses, ratings of the ramp and hold stimuli were averaged across the four stimulus temperatures to yield a mean rating for each participant and for each site of stimulation. These mean ratings were used in subsequent analyses. Associations among the QST measures were assessed via Pearson correlations (Table 2). Correlations between the pain measures were all statistically reliable and of moderate magnitude. This sample was $70 \%$ female, and sex differences in the relationships among pain and psychological measures were conducted with $z$-tests for differences among correlations. No sex differences were found to be reliable $(P>0.05)$. The remainder of the analyses therefore used the entire sample of participants.

Results of the repeated measures ANOVA indicated a significant effect for site of stimulation and supra-threshold response $\left(F(2,238)=20.3, P=0.001\right.$, eta $\left.^{2}=0.15\right)$. Simple contrasts indicated that pain ratings for the lower extremity were lower compared to trunk and upper extremity ratings

Table I Descriptive statistics

\begin{tabular}{ll}
\hline Variable & Pooled $(\mathbf{n}=\mathbf{~ I 2 0})$ \\
\hline Age (years) & $23.6(3.2)$ \\
Sex (\# female) & $70 \%(84 /$ I20) \\
Education (years) & $16.3(1.7)$ \\
Fear (0-I00VAS) & $25.7(18.0)$ \\
Anxiety (0-I00VAS) & $25.7(20.7)$ \\
Thermal threshold (C) & $43.7(3.4)$ \\
Ramp and hold leg (0-100 NRS) & $24.3(19.2)$ \\
Ramp and hold trunk (0-100 NRS) & $31.5(21.5)$ \\
Ramp and hold arm (0-I00 NRS) & $27.3(18.7)$ \\
Wind up leg (0-I00 NRS) & $39.7(28.3)$ \\
Wind up trunk (0-I00 NRS) & $62.7(23.4)$ \\
Wind up arm (0-I00 NRS) & $43.7(27.5)$ \\
After-sensations leg (0-100 NRS) & $12.9(16.4)$ \\
After-sensations trunk (0-I00 NRS) & $18.8(21.4)$ \\
After-sensations arm (0-I00 NRS) & $12.8(15.4)$ \\
\hline
\end{tabular}

Notes: All values reported as mean $( \pm S D)$ or \% (\#).

Abbreviations: VAS, visual analog scale; NRS, numerical rating scale; SD, standard deviation. Ramp and hold reported at $47^{\circ} \mathrm{C}$ only. 
Table 2 Correlations among psychophysical measures

\begin{tabular}{lllllll}
\hline & TS-LE & TS-TR & TS-UE & AS-LE & AS-TR & AS-UE \\
\hline RH-LE & $0.6 I^{* *}$ & $0.70^{* *}$ & $0.56^{* *}$ & $0.53^{* *}$ & $0.59 * *$ & $0.44^{* *}$ \\
RH-TR & $0.62^{* *}$ & $0.8 I^{* *}$ & $0.63^{* *}$ & $0.50^{* *}$ & $0.60^{* *}$ & $0.42^{* *}$ \\
RH-UE & $0.67^{* *}$ & $0.76^{* *}$ & $0.64 * *$ & $0.52^{* *}$ & $0.58^{* *}$ & $0.47^{* *}$ \\
TS-LE & & & & $0.67^{* *}$ & $0.46 * *$ & $0.45^{* *}$ \\
TS-TR & & & & $0.54 * *$ & $0.53^{* *}$ & $0.52^{* *}$ \\
TS-UE & & & & $0.54^{* *}$ & $0.47 * *$ & $0.66 * *$ \\
\hline
\end{tabular}

Note: $* * p<0.01$.

Abbreviations: $\mathrm{RH}$, ramp and hold (supra-threshold response); TS, temporal summation; AS, after-sensation; LE, lower extremity;TR, trunk; UE, upper extremity.

$(P<0.05)$. Upper extremity ratings were also significantly lower than those for the trunk $(P<0.05)$. Results of the repeated measures ANOVA indicated significantly greater temporal summation for the trunk than either lower or upper extremity sites $\left(\mathrm{F}(2,118)=63.2, P<0.001\right.$, eta $\left.^{2}=0.35\right)$. Results of the repeated measures ANOVA indicated a significant effect for site of stimulation and after-sensation $\left(\mathrm{F}(2,238)=11.8, P<0.001\right.$, eta $\left.^{2}=0.09\right)$. Simple contrasts indicated that after-sensation in the trunk was significantly higher than in the lower and upper extremities $(P<0.05)$. However, after-sensation did not differ between lower and upper extremities $(P>0.05)$.

Pearson correlations for the psychological measures are presented in Table 3 and correlations between psychological and QST measures are presented in Table 4. Results of the regressions of fear and anxiety measures are summarized in Table 5. Both the zero-order correlations and the multicollinearity diagnostics indicated there were no violations of multicollinearity assumptions. Furthermore, all variables met criteria for normality (skewness and kurtosis).

For supra-threshold responses, the psychological measures were significant predictors of pain ratings at all anatomical sites with fear of pain scores as a unique predictor.

Table 3 Correlations among psychological measures

\begin{tabular}{llllll}
\hline & Fear-VAS & Anx-VAS & STAI & FPQ & CAT \\
\hline Fear-VAS & & & & & \\
Anx-VAS & $0.79 *$ & & & & \\
STAI & 0.08 & 0.14 & & & \\
FPQ & $0.20^{*}$ & $0.25^{*}$ & $0.26^{*}$ & & \\
CAT & $0.32^{*}$ & $0.31^{*}$ & 0.19 & $0.47^{*}$ & \\
ASI & $0.34^{*}$ & $0.49^{*}$ & $0.24^{*}$ & $0.46^{*}$ & $0.26^{*}$
\end{tabular}

Note: $* P<0.05$.

Abbreviations: Fear-VAS, Fear Visual Analog Scale; Anx-VAS, Anxiety Visual Analog Scale; STAI, Spielberger State-TraitAnxiety Inventory; FPQ, Fear of Pain Questionnaire; CAT + Catastrophizing Scale;ASI + Anxiety Sensitivity Index.
Anxiety sensitivity scores also predicted pain ratings for the lower and upper extremity. All relationships were in the positive direction indicating greater scores on fear of pain and anxiety sensitivity were related to greater pain sensitivity.

For temporal summation, only the regression for the trunk site was statistically reliable $\left(R^{2}=0.29, P=0.004\right)$. Examination of the standardized beta weights indicated that anxiety sensitivity was the only unique contributor and indicated that increased anxiety sensitivity was associated with greater temporal summation. None of the psychological measures were significant predictors of lower extremity temporal summation. Though the overall regression for upper extremity was not statistically reliable, the individual beta weight for anxiety sensitivity was significant and in the same direction as that for trunk site temporal summation.

For after-sensations, psychological measures were statistically reliable predictors of pain sensitivity at the trunk. Examination of standardized beta coefficients indicated that only fear of pain was a unique predictor of after-sensations at the trunk. Neither lower nor upper extremity regressions yielded statistically reliable prediction equations for after-sensations.

\section{Discussion}

QST of individuals with and without pain conditions is increasingly common in the pain literature. There is a wide variety of types of stimulation, sites of stimulation, and stimulation methods employed across investigations. This study contributes to the experimental pain literature and suggests that some of the inconsistencies observed in the literature may be due to the differences related to site of stimulation, type of stimulus procedure, and psychological influence on pain sensitivity.

The first aim of this study was to examine the intercorrelations among the QST measures of supra-threshold response, temporal summation, and after-sensation. These inter-correlations suggest that temporal summation, aftersensation and supra-threshold response, share moderate levels of variance within and across stimulation sites. There was no significant support that presumed C-fiber-related parameters would more closely correlate with each other versus presumed A-delta parameters. Rather, the similar correlations among all psychophysical parameters suggest that an overall pain sensitivity is reflected in the pain responses used in this study. This is somewhat unexpected in a sample of asymptomatic healthy controls. It would be more consistent with a clinical sample that experienced a generalized 
Table 4 Correlations among psychophysical measures

\begin{tabular}{llllllllll}
\hline & TS-LE & TS-TR & TS-UE & AS-LE & AS-TR & AS-UE & RH-LE & RH-TR & RH-UE \\
\hline Fear-VAS & 0.08 & -0.08 & 0.07 & 0.06 & 0.06 & -0.03 & 0.17 & 0.05 & 0.06 \\
Anx-VAS & $0.20^{*}$ & 0.17 & 0.05 & 0.15 & 0.11 & 0.04 & $0.22^{*}$ & 0.17 & $0.18^{*}$ \\
STAI & 0.02 & -0.02 & -0.12 & -0.05 & 0.03 & -0.15 & $0.3^{*}$ & 0.11 & 0.20 \\
FPQ & $0.32^{* *}$ & $0.38^{* *}$ & $0.23^{*}$ & $0.29^{* *}$ & $0.43^{* *}$ & $0.23^{*}$ & $0.55^{* *}$ & $0.5 I^{* *}$ & $0.47^{* *}$ \\
CAT & 0.24 & $0.28^{*}$ & 0.01 & $0.28^{*}$ & $0.34^{* *}$ & 0.04 & $0.31^{*}$ & $0.32^{*}$ & 0.17 \\
ASI & $0.27^{*}$ & $0.30^{*}$ & $0.47^{* *}$ & 0.19 & $0.32^{*}$ & 0.16 & $0.53^{* *}$ & $0.45^{* *}$ & $0.47^{* *}$ \\
\hline
\end{tabular}

Notes: $* P<0.05, * * P<0.01$.

Abbreviations: RH, ramp and hold (supra-threshold response);TS, temporal summation; AS, after-sensation; LE, lower extremity; TR, trunk; UE, upper extremity; Fear-VAS, Fear Visual Analog Scale; Anx-VAS, Anxiety Visual Analog Scale; STAI, Spielberger State-Trait Anxiety Inventory; FPQ, Fear of Pain Questionnaire; CAT + Catastrophizing Scale; $\mathrm{ASI}+$ Anxiety Sensitivity Index.

central sensitization to somatic and painful stimulation. It is plausible that the findings in healthy controls simply reflect the lower range, or normal distribution of central influences on somatic perception.

The second aim of the study was to examine the relationship of site of stimulation and pain responding within and between types of QST. Supra-threshold, after-sensation, and temporal summation were influenced by site of stimulation with a similar pattern. Specifically, all of the sensory parameters showed that the trunk region had higher pain sensitivity, in comparison to the extremities. Our results suggest that the site of stimulation is important to consider when comparing or designing studies. Specifically, ratings from the extremities and the trunk may not be comparable and could result in conclusions that are not robust and indeed may be misleading.

The site of stimulation results may also have implications for neuraxis differences in processing standard pain stimuli for healthy individuals. First, given the high prevalence of chronic low back pain, it seems relevant to note that the trunk had the highest pain sensitivity across all parameters. In a nonpain sample, we did not expect the trunk to be more sensitive to thermal stimulation, but our data suggest the trunk may have elevated pain sensitivity for temporal summation and after-sensation, even in healthy individuals. Furthermore, the measures of temporal summation, in particular, were expected to be more intense in the extremities because of the greater conduction distance compared to the trunk. This finding could indicate that the specific protocol used in the study did not provide sufficient discrimination between A-delta and C-fiber mediated pain responding and that from the trunk; a more blended innervation was experienced.

The final aim of the study was to examine the relationship of QST measures with common psychological constructs (ie, fear, anxiety, and catastrophizing) used in fear-avoidance models of pain. Interestingly, the validated questionnaires measuring trait constructs had the stronger associations with the QST measures, in comparison to state specific VAS ratings of fear and anxiety regarding the about to be experienced stimuli. When associations with fear and anxiety measures were examined, it was revealed that fear of pain and anxiety sensitivity measures were strong predictors of supra-threshold response for all anatomical areas. These data are consistent with some of our previous studies suggesting that fear is associated with experimental pain sensitivity during cold-pressor for healthy participants, ${ }^{29,30}$ and thermal pain for participants with low back pain. ${ }^{31}$ Fear of pain and anxiety sensitivity measures were consistently associated with trunk pain perception for all three parameters (ie, after sensation, temporal summation, and supra-threshold stimulation), but not with lower and upper extremity pain perception. This finding was counter to one of our earlier studies suggesting anxiety was associated with temporal summation in the upper extremity. ${ }^{32}$ Previous studies have not investigated psychological influence on after-sensation, so there are few comparative studies available. Collectively these data indicate that psychological influence might be more consistent across sites for A-delta fiber mediated processes (supra-threshold response), with more site-specific (trunk) influence on purported $\mathrm{C}$-fiber processes (temporal summation and after-sensation).

Limitations of the study include restricted generalizability to clinical populations. The use of asymptomatic control participants with presumably normally functioning nervous systems may mean that the results are not compatible with individuals whose chronic pain has altered their perception of normally, nonpainful and painful stimulation through central sensitization processes. Furthermore, the experience of chronic pain may also alter the expectations and associations 
Table 5 Results of psychological predictors of pain measures

\begin{tabular}{|c|c|c|c|c|c|}
\hline $\begin{array}{l}\text { Psychophysical } \\
\text { measure }\end{array}$ & Site & $R^{2}$ & $\boldsymbol{F}$ & $p$ & $\beta$ \\
\hline \multirow[t]{21}{*}{$\mathrm{RH}$} & LE & 0.45 & 70.2 & 0.002 & \\
\hline & & & & & $\mathrm{FPQ}=0.32^{*}$ \\
\hline & & & & & $\mathrm{ASI}=0.29 *$ \\
\hline & & & & & FearVAS $=0.20$ \\
\hline & & & & & AnxVAS $=-0.03$ \\
\hline & & & & & $S T A I=0.13$ \\
\hline & & & & & CAT $<0.0 \mathrm{I}$ \\
\hline & $\mathrm{TR}$ & 0.32 & 40.2 & 0.002 & \\
\hline & & & & & $\mathrm{FPQ}=0.33^{*}$ \\
\hline & & & & & $\mathrm{ASI}=0.27$ \\
\hline & & & & & FearVAS $=-0.13$ \\
\hline & & & & & AnxVAS $=0.13$ \\
\hline & & & & & $\mathrm{STAI}=-0.06$ \\
\hline & & & & & $\mathrm{CAT}=0.10$ \\
\hline & UE & 0.31 & 30.9 & 0.003 & \\
\hline & & & & & $\mathrm{FPQ}=0.3 \mathrm{I}^{*}$ \\
\hline & & & & & $\mathrm{ASI}=0.28^{*}$ \\
\hline & & & & & FearVAS $=-0.08$ \\
\hline & & & & & $A n x V A S=0.15$ \\
\hline & & & & & STAI $=0.07$ \\
\hline & & & & & CAT $=-0.09$ \\
\hline
\end{tabular}

$\begin{array}{lllll}\text { TS } & \text { LE } & 0.12 & 0.47 & >0.05\end{array}$

AS

LE $\quad 0.15 \quad 10.6 \quad>0.05$

$\mathrm{FPQ}=0.05$

$\mathrm{ASI}=0.18$

FearVAS $=-0.11$

AnxVAS $=0.15$

$\mathrm{STAI}=-0.08$

$\mathrm{CAT}=0.18$

$\begin{array}{llll}\text { TR } & 0.29 & 30.7 & 0.004\end{array}$

$\mathrm{FPQ}=0.12$

$\mathrm{ASI}=0.36^{*}$

FearVAS $=-0.14$

AnxVAS $=0.22$

$\mathrm{STAI}=-0.18$

CAT $=0.14$

$\begin{array}{llll}\text { UE } & 0.15 & 10.6 & 0.160\end{array}$

$\mathrm{FPQ}=0.09$

$\mathrm{ASI}=0.33^{*}$

FearVAS $=-0.23$

AnxVAS $=0.13$

STAI $=-0.24$

$\mathrm{CAT}=-0.05$
Table 5 (Continued)

$$
\begin{aligned}
& \text { ASI }=0.07 \\
& \text { FearVAS }=0.01 \\
& \text { AnxVAS }=0.01 \\
& \text { STAI }=-0.16 \\
& \text { CAT }=0.17 \\
& \text { FPQ }=0.36^{*} \\
& \text { ASI }=0.16 \\
& \text { FearVAS }=0.07 \\
& \text { AnxVAS }=-0.09 \\
& \text { STAI }=-0.12 \\
& \text { CAT }=0.16 \\
& \text { FPQ }=0.10 \\
& \text { ASI }=0.16 \\
& \text { FearVAS }=-0.10 \\
& \text { AnxVAS }=0.09 \\
& \text { STAI }=-0.20 \\
& \text { CAT }<00.01
\end{aligned}
$$

$\begin{array}{llll}\text { TR } & 0.26 & 30.2 & 0.010\end{array}$

UE $\quad 0.07 \quad 0.68 \quad>0.05$

Note: $* P<0.05$.

Abbreviations: $\mathrm{RH}$, ramp and hold (supra-threshold response); TS, temporal summation; AS, after-sensation; LE, lower extremity; TR, trunk; UE, upper extremity.

$\mathrm{FPQ}=0.24$

(Continued) between psychological measures and the experience of pain in both quantitative and qualitative processes. The level of psychological measures is likely to be much lower in this asymptomatic sample than in a chronic pain sample. The generalizability of these findings to different psychophysical protocols, and different methods of inducing supra-threshold, temporal summation, and after-sensation pain may also be limited. Finally, our psychological measures were not stimulation-site specific. We can only speculate about differences in anxiety sensitivity and fear related to trunk versus extremity associations to pain stimulation. Perhaps stimulation to the trunk is more novel, and elicits greater anxiety and fear than extremity stimulation, resulting in greater associations between pain and psychological measures at the trunk. Future studies might be improved by including site-specific measures of anxiety and fear.

In summary, this was one of the first studies to directly compare different QST induction methods within the same individual and across body sites. Results suggest that previous assumptions of $\mathrm{A}$-delta and $\mathrm{C}$-fiber mediated pain processes may not be adequately reflected in the described QST protocol. Furthermore, site of stimulation may be an important consideration when designing and comparing 
studies using these methods. For both supra-threshold response and after-sensation pain ratings differed by site; with trunk stimulation generally experienced as more intense. Supra-threshold response was highly associated with fear and anxiety measures across all anatomical sites, while temporal summation and after-sensations were only correlated with anxiety sensitivity and fear at the trunk respectively. Temporal summation ratings were higher for trunk stimulation and consistently associated with anxiety sensitivity at the same site. Therefore, studies designed to examine pain-related fear and anxiety sensitivity are more likely to find associations if they employ supra-threshold response methods at most sites of stimulation, although temporal summation associations appeared stronger with trunk stimulation.

\section{Acknowledgments}

This research was supported by grants AR054331, HD043730, HD055929, and AT002796 from the National Institutes of Health.

\section{Disclosures}

The authors have no conflicts of interest in the conduct or reporting of this research.

\section{References}

1. Siao P, Cros DP. Quantitative sensory testing. Phys Med Rehabil Clin NAm. 2003;14(2):261-286.

2. Petersen KL, Rowbotham MC. Quantitative sensory testing scaled up for multicenter clinical research networks: a promising start. Pain. 2006;123(3):219-220.

3. Rolke R, Baron R, Maier C, et al. Quantitative sensory testing in the German Research Network on Neuropathic Pain (DFNS): standardized protocol and reference values. Pain. 2006;123(3):231-243.

4. Hansson P, Backonja M, Bouhassira D. Usefulness and limitations of quantitative sensory testing: clinical and research application in neuropathic pain states. Pain. 2007;129(3):256-259.

5. Staud R, Robinson ME, Price DD. Temporal summation of second pain and its maintenance are useful for characterizing widespread central sensitization of fibromyalgia patients. J Pain. 2007;8(11):893-901.

6. Staud R, Vierck CJ, Robinson ME, Price DD. Overall fibromyalgia pain is predicted by ratings of local pain and pain-related negative affect - possible role of peripheral tissues. Rheumatology (Oxford). 2006;45(11):1409-1415.

7. Maixner W, Fillingim R, Sigurdsson A, Kincaid S, Silva S. Sensitivity of patients with painful temporomandibular disorders to experimentally evoked pain: evidence for altered temporal summation of pain. Pain. 1998;76(1-2):71-81.

8. George SZ, Bishop MD, Bialosky JE, Zeppieri G Jr, Robinson ME. Immediate effects of spinal manipulation on thermal pain sensitivity: an experimental study. BMC Musculoskelet Disord. 2006;7:68.

9. Hagander LG, Midani HA, Kuskowski MA, Parry GJ. Quantitative sensory testing: effect of site and pressure on vibration thresholds. Clin Neurophysiol. 2000;111(6):1066-1069.

10. Gaskin ME, Greene AF, Robinson ME, Geisser ME. Negative affect and the experience of chronic pain. J Psychosom Res. 1992;36(8): 707-713.
11. Staud R, Robinson ME, Vierck CJ Jr, Cannon RC, Mauderli AP, Price DD. Ratings of experimental pain and pain-related negative affect predict clinical pain in patients with fibromyalgia syndrome. Pain. 2003;105(1-2):215-222.

12. Vlaeyen JW, Linton SJ. Fear-avoidance and its consequences in chronic musculoskeletal pain: a state of the art. Pain. 2000;85(3):317-332.

13. Leeuw M, Goossens ME, Linton SJ, Crombez G, Boersma K, Vlaeyen JW. The fear-avoidance model of musculoskeletal pain: current state of scientific evidence. J Behav Med. 2007;30(1):77-94.

14. George SZ, Wittmer VT, Fillingim RB, Robinson ME. Fear-avoidance beliefs and temporal summation of evoked thermal pain influence selfreport of disability in patients with chronic low back pain. J Occup Rehabil. 2006;16(1):95-108.

15. Bialosky JE, Bishop MD, Robinson ME, Barabas JA, George SZ. The influence of expectation on spinal manipulation induced hypoalgesia: an experimental study in normal subjects. BMC Musculoskelet Disord. 2008;9:19.

16. Schmidt NB, Joiner TE. Structure of the Anxiety Sensitivity Index psychometrics and factor structure in a community sample. J Anxiety Disord. 2002;16(1):33-49.

17. Dehon C, Weems CF, Stickle TR, Costa NM, Berman SL. A crosssectional evaluation of the factorial invariance of anxiety sensitivity in adolescents and young adults. Behav Res Ther. 2005;43(6): 799-810.

18. Spielberger CD, Gorsuch RL, Lushene RE, Vagg PR, Jacobs GA. Manual for the State Trait Anxiety Inventory (Form Y). Palo Alto, CA: Consulting Psychologists Press; 1983.

19. Rosenstiel AK, Keefe FJ. The use of coping strategies in chronic low back pain patients: relationship to patient characteristics and current adjustment. Pain. 1983;17(1):33-44.

20. Robinson ME, Riley JL 3rd, Myers CD, et al. The Coping Strategies Questionnaire: a large sample, item level factor analysis. Clin J Pain. 1997;13(1):43-49.

21. McNeil DW, Rainwater AJ 3rd. Development of the Fear of Pain Questionnaire - III. J Behav Med. 1998;21(4):389-410.

22. Albaret MC, Munoz Sastre MT, Cottencin A, Mullet E. The Fear of Pain questionnaire: factor structure in samples of young, middle-aged and elderly European people. Eur J Pain. 2004;8(3):273-281.

23. Osman A, Breitenstein JL, Barrios FX, Gutierrez PM, Kopper BA. The Fear of Pain Questionnaire-III: further reliability and validity with nonclinical samples. J Behav Med. 2002;25(2):155-173.

24. Price DD, Hu JW, Dubner R, Gracely RH. Peripheral suppression of first pain and central summation of second pain evoked by noxious heat pulses. Pain. 1977;3(1):57-68.

25. Fillingim RB, Ness TJ, Glover TL, Campbell CM, Price DD, Staud R. Experimental pain models reveal no sex differences in pentazocine analgesia in humans. Anesthesiology. 2004;100(5): 1263-1270.

26. Staud R, Craggs JG, Robinson ME, Perlstein WM, Price DD. Brain activity related to temporal summation of $\mathrm{C}$-fiber evoked pain. Pain. 2007;129(1-2):130-142.

27. Staud R, Craggs JG, Perlstein WM, Robinson ME, Price DD. Brain activity associated with slow temporal summation of $\mathrm{C}$-fiber evoked pain in fibromyalgia patients and healthy controls. Eur J Pain. 2008;12(8):1078-1089.

28. Staud R, Vierck CJ, Cannon RL, Mauderli AP, Price DD. Abnormal sensitization and temporal summation of second pain (wind-up) in patients with fibromyalgia syndrome. Pain. 2001;91(1-2): 165-175.

29. George SZ, Dannecker EA, Robinson ME. Fear of pain, not pain catastrophizing, predicts acute pain intensity, but neither factor predicts tolerance or blood pressure reactivity: an experimental investigation in pain-free individuals. Eur J Pain. 2006;10(5): 457-465.

30. Hirsh AT, George SZ, Bialosky JE, Robinson ME. Fear of pain, pain catastrophizing, and acute pain perception: relative prediction and timing of assessment. J Pain. 2008;9(9):806-812. 
31. George SZ, Wittmer VT, Fillingim RB, Robinson ME. Sex and painrelated psychological variables are associated with thermal pain sensitivity for patients with chronic low back pain. J Pain. 2007;8(1):2-10.
32. Robinson ME, Wise EA, Gagnon C, Fillingim RB, Price DD. Influences of gender role and anxiety on sex differences in temporal summation of pain. J Pain. 2004;5(2):77-82.

\section{Publish your work in this journal}

The Journal of Pain Research is an international, peer-reviewed, open access, online journal that welcomes laboratory and clinical findings in the fields of pain research and the prevention and management of pain. Original research, reviews, symposium reports, hypothesis formation and commentaries are all considered for publication.
Dovepress

The manuscript management system is completely online and includes a very quick and fair peer-review system, which is all easy to use. Visit http://www.dovepress.com/testimonials.php to read real quotes from published authors. 